\title{
Call for Papers: Special Issue of Earth, Planets and Space (EPS) "Cosmic Dust: Its Formation and Evolution (II)"
}

This issue of EPS will be devoted to the session "Cosmic Dust: Its Formation and Evolution" of the AOGS 2010 meeting held in Hyderabad, India. It is meant primarily to contain the papers presented at this particular session of AOGS 2010, but it is also open to submission of any other papers, following the regular EPS submission procedures. Contributions should discuss any aspect of cosmic dust. All kinds of cosmic dust such as intergalactic dust, interstellar dust, circumstellar dust, cometary dust, asteroidal dust, interplanetary dust, circumplanetary dust, stellar nebular condensates, presolar grains, micrometeorites, meteoroids, meteors, and regolith particles are the subject of discussion.

All submitted papers go through a peer review process, wherein experts review, recommend, or reject the papers for publishing. Submitted papers should not have been previously published nor be currently under consideration for publication elsewhere. Papers must be submitted to the EPS editorial office. Electronic submissions by e-mail to eps@terrapub.co.jp are encouraged, but authors who meet any difficulties with electronic submissions could submit a hard copy by post. For details, please visit the following web page:

http://www.terrapub.co.jp/journals/EPS and click on "Information for Contributors".

The cover letter should mention "Submitted to the Special Issue: Cosmic Dust (II)". After papers are accepted, the authors will receive instructions for the final manuscript from the editorial office. The deadline for manuscript submissions is 15 September 2010. This special issue will hopefully be published in June 2011.

For more information on this special issue, please contact the corresponding editor H. Kimura (hiroshi@kitty.kobe-u.ac.jp); questions on manuscript preparation should be addressed to the EPS editorial office.

Note: EPS accepts manuscripts of original research contributions only, and so-called "review papers" will not be accepted.

Guest Editors: Hiroshi Kimura, Center for Planetary Science, Japan

Misato Fukagawa, Osaka University, Japan

Cornelia Jäger, Friedrich Schiller University, Germany

Ludmilla Kolokolova, University of Maryland, USA

Aigen Li, University of Missouri-Columbia, USA

Keiko Nakamura-Messenger, NASA Johnson Space Center, USA 